\title{
A Collision Model for Protective Structure with Gradient Metallic Cellular Material under Low Velocity Impact
}

\author{
Dengbao Xiao and Guiping Zhao \\ State Key Laboratory for Strength and Vibration of Mechanical Structures, School of Aerospace, Xian Jiaotong University, \\ Xian 710049, China \\ Correspondence should be addressed to Guiping Zhao; zhaogp@mail.xjtu.edu.cn
}

Received 19 May 2016; Revised 27 July 2016; Accepted 15 August 2016

Academic Editor: Mickaël Lallart

Copyright (C) 2016 D. Xiao and G. Zhao. This is an open access article distributed under the Creative Commons Attribution License, which permits unrestricted use, distribution, and reproduction in any medium, provided the original work is properly cited.

During quasi-static compressive process, the gradient metallic cellular material can be divided into deformed region and undeformed region before the material is compressed compactly. The deformation mode of the gradient metallic cellular material is not the same as that of homogeneous metallic cellular material. Meanwhile, no plateau stress is observed in the crushing stress versus strain curve of the gradient metallic cellular material. A formula of calculating crushing stress of the gradient metallic cellular material is presented. Subsequently, a collision model for protective structures with gradient metallic cellular material is presented to investigate the vibration isolation of the large mass protection under low velocity impact based on Lagrange's equation of the second kind. The effects of the gradient metallic cellular material on the acceleration peak of inner protected structure are theoretically discussed. And the results show that adopting gradient metallic cellular material instead of homogeneous metallic cellular material with equivalent mass can decrease the peak acceleration value of the inner protected structure.

\section{Introduction}

Because of the outstanding properties, such as the low weight and high efficient energy absorption $[1,2]$, the metallic cellular materials have been extensively applied in the civilian and aerospace fields (e.g., reentry capsule and airdrop platform) for impact mitigation applications. Among the metallic cellular materials, the gradient metallic cellular material has attracted many research interests $[3,4]$. For the gradient metallic cellular material, its pore structures were graded through the thickness direction of the material resulting in varying mechanical properties. Meanwhile, the variation of the mechanical property may considerably influence its impact mitigation performances. Thus, the influence of the gradient metallic cellular material on the dynamic response of the protected structures is interesting [5-7].

The compressive properties of the gradient metallic cellular material are important to its impact resistance performances under impact loading. Ali et al. [8] investigated the crushing stress of the gradient hexagonal structure subjected to the low velocity impact. Their results showed that the deformation mode of the gradient hexagonal structure was
"I" mode [9]. In "I" mode, compressing deformation began from the part of the gradient cellular material with the minimum plateau stress. And the deforming process carried on until the part of the gradient cellular material with the maximum plateau stress is crushed. Ajdari et al. [10] constructed the finite element (FE) model of the gradient Voronoi cellular structure and investigated its uniaxial deformation behavior under the quasi-static compression. The results also suggested the deformation mode was "I" mode. Hangai et al. [11] fabricated the gradient aluminum foam and stated that it had exhibited multiple plateau stresses. $\mathrm{He}$ et al. [12] investigated the effect of gradient structures on mechanical properties of the gradient aluminum foam under quasi-static compression. It was reported that the crushing stress increased with remarkable positive slope in the experimental nominal stress-strain curve of the gradient aluminum foam. Xiao et al. $[13,14]$ presented a simplified formula to calculate the quasi-static crushing stress of the gradient metallic cellular material. The RPPL (rigid-perfectly plastic-locking) model [15] was employed by Xiao et al. to model the metallic cellular material. In views of neglecting the strain hardening in RPPL model, the deformed cellular 


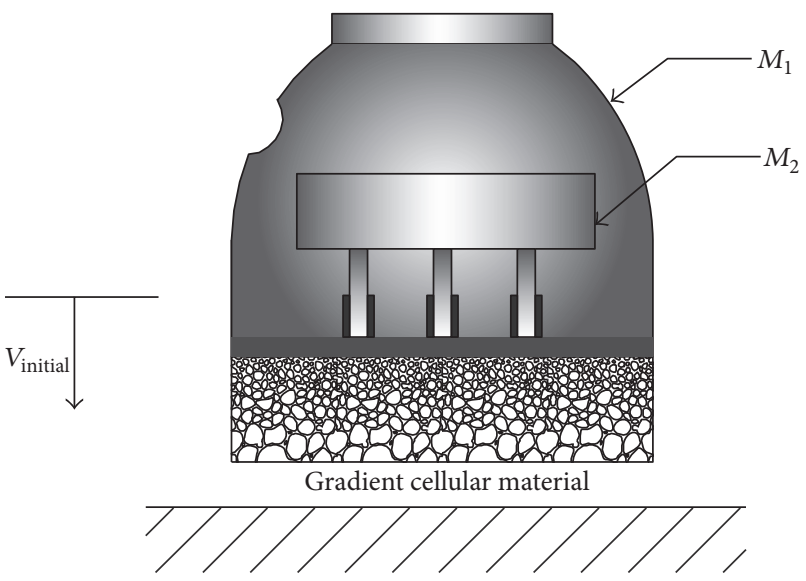

(a)

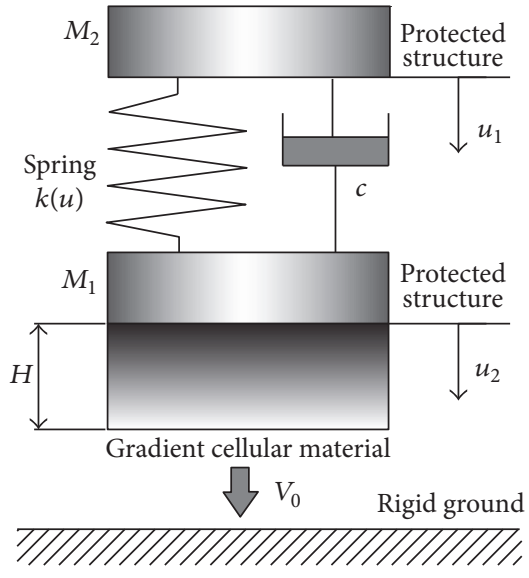

(b)

FIGURE 1: (a) Reentry capsule collides with hard ground; (b) collision model for protective structure with gradient metallic cellular material.

materials have been densified and their strains have reached densification strain during the compression.

In most of previous researches, the metallic cellular materials were used to absorb impact energy to prevent the inner protected structures/components from failure or destruction during collision process [16-19]. When the collision energy was exerted on the outer structure, the dynamic response (vibration isolation) of the inner protected structures/components (seat-occupant system) is an important issue. Joshi et al. [20] used a single degree of freedom massspring-damper model to discuss the vibration isolation of the seat-occupant. The dynamic response of the seated occupant was discussed. Toward and Griffin [21] developed a similar mass-spring-damper model to study the effects of sitting posture and vibration magnitude on the vertical apparent mass of the human body. Li et al. [22,23] presented a double degree of freedom collision model to investigate the dynamic responses of the inner objects and the outer structure protected by metallic foam. The uniaxial compressive stress-strain curve of homogeneous foam was experimentally obtained by $\mathrm{Li}$ et al. [24]. In their studies, the effects of mass/damping ratio and initial impact velocity on peak acceleration of the protected structure were discussed. So far, the energy absorber in protective structure is mostly limited to homogeneous cellular material. Adopting the gradient metallic cellular material for energy absorber instead of homogeneous metallic cellular material in the protective structures, the vibration isolation of the inner protected structure and the outer structure under low velocity impact is an interesting research issue.

The outline of the present paper is as follows. Firstly, a formula considering hardening strain for cellular material is presented to predict the crushing stress of the gradient metallic cellular material. Secondly, based on Lagrange's equation of the second kind, a collision model for protective structure with gradient metallic cellular material is developed for large mass protection structure under low velocity impact.
Finally, the influences of gradient metallic cellular material on the dynamic response of the inner protected structure are investigated.

\section{The Collision Model for Protective Structure with Gradient Metallic Cellular Material under Low Velocity Impacting}

When a reentry space capsule lands on the ground, the cushion material and the seat are the important protection system for the occupant. The process of the reentry capsule colliding with hard ground can be simplified as a low velocity impact problem with a large mass structure protected by the gradient metallic cellular material shown in Figure 1(a). The reentry capsule is simplified as a rigid body having mass $M_{1}$; the occupant and seat together are treated as a rigid structure having mass $M_{2}$. Meanwhile, the seatoccupant structure is modeled as a mass-spring-damper system. Let the spring and damper be characterized by $k(u)$ and $c$, respectively. Therefore, the reentry capsule colliding problem can be modeled as a collision model for protective structure with gradient metallic cellular material as shown in Figure 1(b).

2.1. Quasi-Static Crushing Stress for the Gradient Metallic Cellular Material. The gradient metallic cellular material has an initial mass density that increases from upper face to bottom face shown in Figure 2. $H$ notes the thickness of the gradient metallic cellular material and $z$ is a coordinate of thickness direction. Not considering detailed material topology, the characteristics of gradient cellular materials were simplified as homogeneous in plane and varied along thickness direction. Zheng et al. [25] calculated the quasistatic crushing stress of homogeneous cellular material using an empirical function with plastic strain hardening stage. The empirical function is employed to calculate the quasi-static 


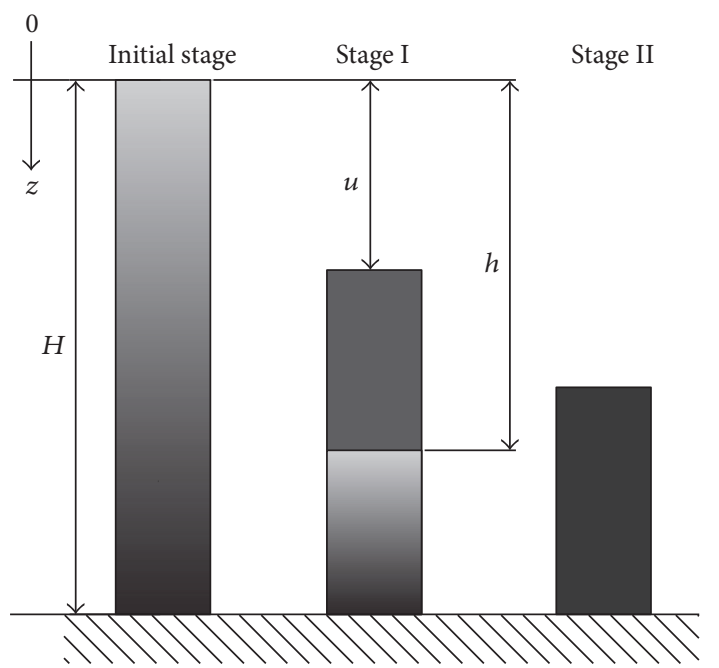

Figure 2: Compressive deformation of the gradient metallic cellular material.

crushing stress of the cellular material with mass densities depending on direction coordinate:

$$
\sigma_{\text {initial }}(z, \varepsilon)=\sigma_{p}(z)+\frac{c(z) \cdot \varepsilon(z)}{(1-\varepsilon(z))^{2}}
$$

where $\sigma_{p}(z), c(z)$, and $\varepsilon(z)$ are the initial plateau stress, strain hardening parameter, and strain function of coordinate $z$, respectively. Based on the investigation [25], Wang et al. [26] presented the relationship between the strain hardening parameter and relative mass density of the cellular material as follows:

$$
c(z)=0.115 \cdot \sigma_{s} \cdot\left(\frac{\rho_{\text {initial }}(z)}{\rho_{s}}\right)^{1.5},
$$

where $\sigma_{s}, \rho_{s}$ and $\rho_{\text {initial }}(z)$ are the yield stress, mass density of the matrix material, and initial linear mass density distribution, respectively. Meanwhile, $\sigma_{p}(z)$ is the initial plateau stress [27]:

$$
\sigma_{p}(z)=\sigma_{s} \cdot\left(\frac{\rho_{\text {initial }}(z)}{\rho_{s}}\right)^{1.5} .
$$

The initial linear mass density distribution of gradient cellular materials is defined as follows:

$$
\rho_{\text {initial }}(z)=\rho_{1}+\frac{\left(\rho_{2}-\rho_{1}\right) \cdot z}{H},
$$

where $\rho_{1}$ and $\rho_{2}$ are the initial mass density of the upper surface and bottom surface, respectively.

Before all metallic cellular materials entered into densification under quasi-static compression in Figure 2, the gradient metallic cellular material is compressed with deformation $u$. In stage I, the gradient cellular material can be divided into deformed materials and undeformed materials. And the compressing deformation $u$ results from the deformed materials with length $h$. In stage II, the cellular materials have been all crushed and the crushing stress increases sharply.
The relationship between the compressing deformation $u$ and deformed region $h$ can be expressed as follows:

$$
u=f(h)=\int_{0}^{h} \varepsilon(z) d z
$$

Owing to quasi-static compression, the stress distribution of gradient cellular material can be considered as homogeneous. Hence, the crushing stress of gradient cellular material is equal to the yield stress of cellular material which begins crushing and can be written as

$$
\sigma_{g c}(u)=\sigma_{\text {initial }}(h, 0) \text {. }
$$

According to (1)-(6), the crushing stress $\sigma_{g c}(u)$ during stage I can be obtained:

$$
\sigma_{g c}(u)=g(u)=g(f(h)), \quad 0 \leq h \leq H,
$$

where $g(u)$ is the implicit function about $u$. Adopting bisection method to solve the above implicit function (7), the relationship of crushing stress and compressing deformation $u$ can be obtained.

In stage II, all cellular materials have been crushed. The crushing stress in stage II can be calculated by the following:

$$
\sigma_{g c}(u)=\sigma\left(H, \varepsilon_{b}\right)
$$

where $\varepsilon_{b}$ is the strain of bottom face.

2.2. Mathematical Formulations for the Collision Model. The method based on Lagrange's equation of the second kind is employed to analyze the dynamic responses of the model. The initial time is set exactly at the moment when the gradient cellular material contacts the rigid ground shown in Figure 1(b). The origins of the coordinates $u_{1}$ and $u_{2}$ for the model are located at the initial positions of $M_{1}$ and $M_{2}$, respectively.

For the model, the Lagrange function $L$ of the system may be defined as

$$
L=K-G-E_{g c}-E_{g s},
$$

where $K$ is the kinetic energy of the system, $G$ is the gravitational potential energy, $E_{g c}$ is the plastic energy of gradient cellular material, and $E_{g s}$ is the elastic potential energy of the spring. Since the mass of the gradient cellular material is typically much lower than that of the protected structure, the kinetic energy of the gradient cellular material is neglected under the present low velocity impact. The total kinetic energy $K$ can be defined as

$$
K=\frac{1}{2} M_{1} \dot{u}_{1}^{2}+\frac{1}{2} M_{2} \dot{u}_{2}^{2},
$$


where $\dot{u}_{1}$ and $\dot{u}_{2}$ are separately the velocities of $M_{1}$ and $M_{2}$. And the gravitational potential energy is expressed as a function of the displacements of $M_{1}$ and $M_{2}$ at time $t$ :

$$
G=-\left(M_{1} g u_{1}+M_{2} g u_{2}\right) \text {. }
$$

During collision, the plastic energy of the gradient cellular material is calculated as follows:

$$
E_{g c}=\int_{0}^{u_{1}} \sigma\left(u_{1}\right) S d u,
$$

where $\sigma\left(u_{1}\right)$ is the crushing stress of the gradient cellular material calculated by (7) and (8) and $S$ is the cross-sectional area of the gradient cellular material. Note that the frictions on the top/bottom gradient cellular material surfaces are neglected. Therefore, the cross-sectional area $S$ can be seen as a constant.

The elastic potential energy of the spring is determined by the displacement difference between $M_{1}$ and $M_{2}$ :

$$
E_{s}=\int_{0}^{u_{2}-u_{1}} k(u) d u
$$

where $k(u)$ is the stiffness of spring varying with the difference between $u_{1}$ and $u_{2}$. And $k(u)$ can be simplified and defined as

$$
\begin{gathered}
k(u)=k \cdot \Delta u+k_{0}, \\
\Delta u=u_{1}-u_{2},
\end{gathered}
$$

where $k_{0}$ is the initial stiffness value and $k$ is the coefficient.

Substituting (14a) and (14b) into (13), the elastic potential energy of the spring is obtained as

$$
E_{s}=\frac{1}{3} k\left(u_{2}-u_{1}\right)^{3}+\frac{1}{2} k_{0}\left(u_{2}-u_{1}\right)^{2}
$$

The viscous damping force varied linearly with the relative velocity as follows:

$$
p_{d}=C\left(\dot{u}_{2}-\dot{u}_{1}\right) \text {, }
$$

where $C$ is the damping coefficient.

Therefore the damping dissipated work is calculated by

$$
W_{d}=\int_{0}^{u_{2}-u_{1}} p_{d} d u=\int_{0}^{u_{2}-u_{1}} c\left(\dot{u}_{2}-\dot{u}_{1}\right) d u .
$$

The work dissipated by friction is neglected. Hence, the total work of the system is equal to those of damp:

$$
W=W_{d}
$$

Accordingly, the generalized nonpotential forces $Q_{1}$ and $Q_{2}$ are obtained as follows:

$$
Q_{i}=\frac{\partial W}{\partial u_{i}} \quad(i=1,2)
$$

where $W$ is the dissipated work of the system. Substituting (18) into (19), the nonpotential force can be rewritten as

$$
\begin{aligned}
& Q_{1}=\frac{\partial W}{\partial u_{1}}=c\left(\dot{u}_{2}-\dot{u}_{1}\right), \\
& Q_{2}=\frac{\partial W}{\partial u_{2}}=-c\left(\dot{u}_{2}-\dot{u}_{1}\right) .
\end{aligned}
$$

Based on Lagrange's equation of the second kind,

$$
\frac{d}{d t} \frac{\partial L}{\partial \dot{u}_{i}}-\frac{\partial L}{\partial u_{i}}=Q_{i} \quad(i=1,2) ;
$$

the equilibrium equations of the model are derived by substituting (10)-(13), (20a), and (20b) into (21) as

$$
\begin{aligned}
& M_{1} \ddot{u}_{1}-M_{1} g+\sigma(u) S-k\left(u_{2}-u_{1}\right)^{2}-k_{0}\left(u_{2}-u_{1}\right) \\
& \quad=Q_{1}, \\
& M_{2} \ddot{u}_{2}-M_{2} g+k\left(u_{2}-u_{1}\right)^{2}+k_{0}\left(u_{2}-u_{1}\right)=Q_{2} .
\end{aligned}
$$

The initial conditions are

$$
\begin{aligned}
& \left.u_{1}(t)\right|_{t=0}=\left.u_{2}(t)\right|_{t=0}=0, \\
& \left.\dot{u}_{1}(t)\right|_{t=0}=\left.\dot{u}_{2}(t)\right|_{t=0}=V_{0} .
\end{aligned}
$$

The explicit integral method is employed to solve (22a), (22b), and (23).

\section{Verification of the Collision Model for Protective Structure with Gradient Metallic Cellular Material}

3.1. Verification of the Crushing Stress of the Gradient Metallic Cellular Material. A numerical compressive "test" of the gradient metallic cellular material at a low constant velocity ( $V=0.5 \mathrm{~m} / \mathrm{s}$ ) was performed by using ABAQUS to obtain the crushing stress and then the results were compared with those calculated by presented model. In the simulation, the axisymmetric FE models of the gradient metallic cellular cylinder and a rigid plate are constructed. The radius of the cylinder is $10 \mathrm{~mm}$, and the thickness is $20 \mathrm{~mm}$. The sample is divided into 20 layers along thickness direction, and the thickness of each layer is the same. The sample is simulated by a 4-node bilinear axisymmetric quadrilateral CAX4R. Each layer contains 400 elements. The constitutive law "crushable foam" with isotropic hardening in the ABAQUS/EXPLICIT is employed to present the properties of the sample. Meanwhile, the "velocity boundary condition" is used to control the velocity of the rigid plate. The bottom of the sample is fixed. The matrix material of the cellular sample is aluminum and its plastic Poisson ratio is 0 .

The corresponding mass densities are as follows: $\rho_{1}=$ $270 \mathrm{~kg} / \mathrm{m}^{3}$ (relative mass density $\overline{\rho_{1}}=0.1$ ) and $\rho_{2}=$ $810 \mathrm{~kg} / \mathrm{m}^{3}$ (relative mass density $\overline{\rho_{2}}=0.3$ ). The quasi-static stress-strain curve $((7)$ and (8)) of gradient cellular sample under quasi-static compression is shown in Figure 3. 


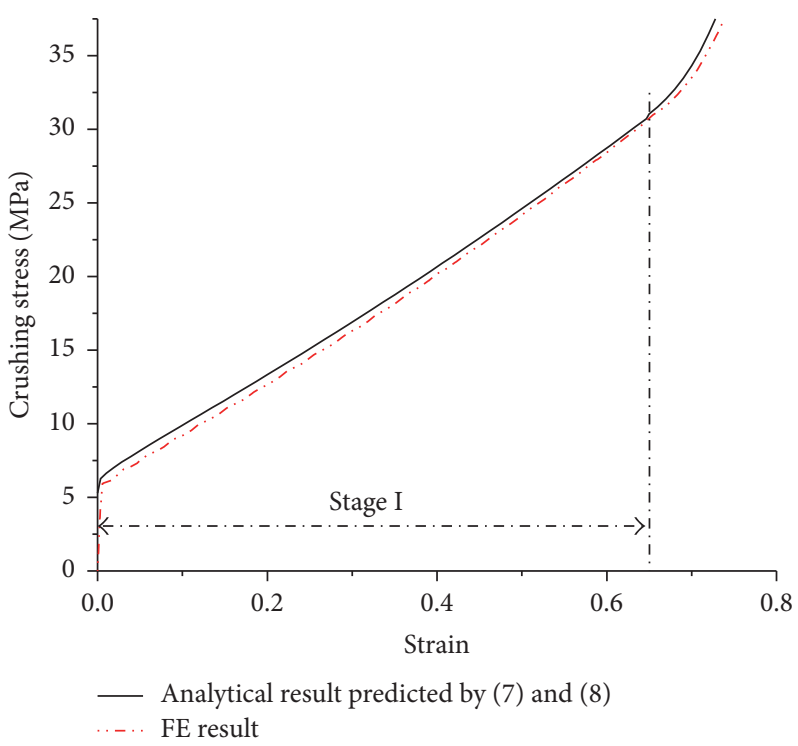

FIgURE 3: The quasi-static stress-strain curves of gradient cellular material obtained from the FE model and the theoretical prediction.

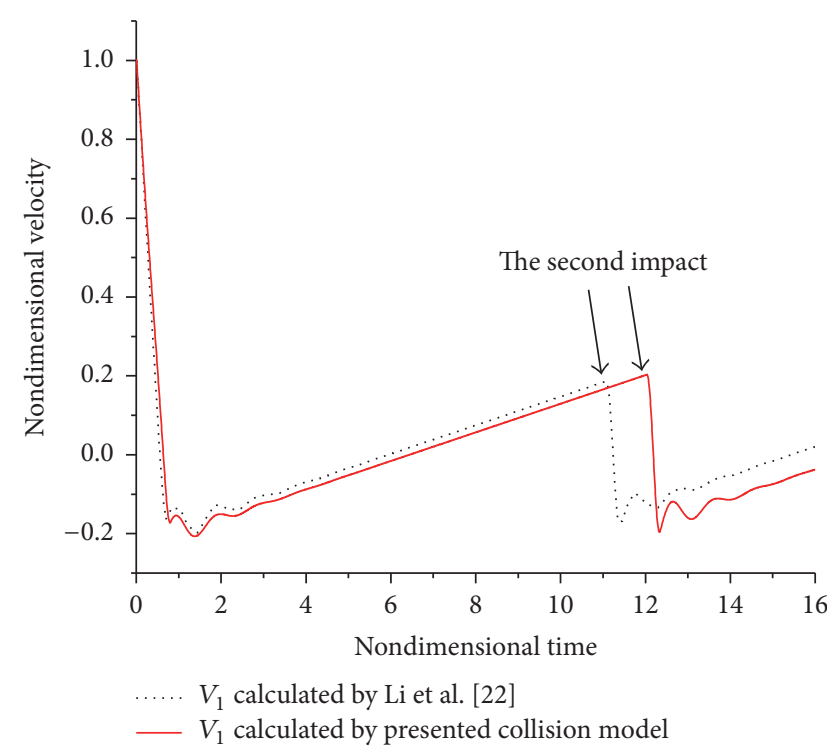

(a)

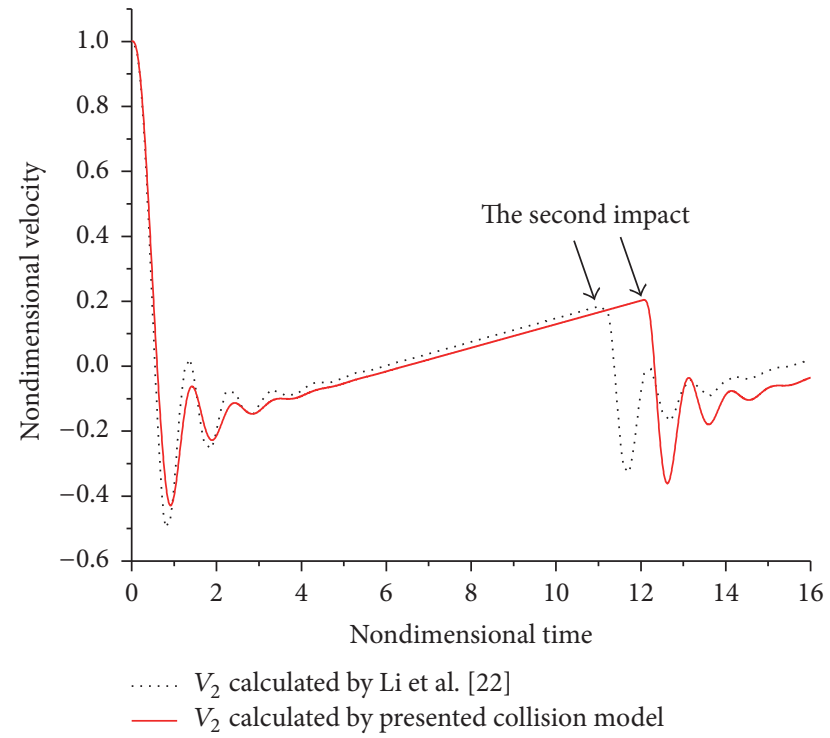

(b)

FIGURE 4: The velocity attenuation predicted by presented collision model: (a) outer protected structure $M_{1}$ and (b) inner protected structure $M_{2}$.

In Figure 3, the crushing stress-nominal strain curve considering the strain hardening for cellular material is very close to FE results. When the nominal strain exceeds 0.65 , the gradient cellular sample has all been crushed.

3.2. Verification of the Collision Model for Protective Structure with Gradient Metallic Cellular Material. To validate the presented collision model, the mass density of the gradient cellular material is taken as $\rho_{1}=\rho_{2}=327.2 \mathrm{~kg} / \mathrm{m}^{3}$ in (4). The present collision model will degenerate into the collision model for protective structure with homogeneous cellular material. Figure 4 shows the velocity attenuation of
TABle 1: Physical parameters used in the collision model.

\begin{tabular}{lcccccc}
\hline$M_{1}(\mathrm{Kg})$ & $M_{2}(\mathrm{Kg})$ & $k\left(\mathrm{~N} / \mathrm{m}^{2}\right)$ & $k_{0}(\mathrm{~N} / \mathrm{m})$ & $H(\mathrm{~m})$ & $S\left(\mathrm{~m}^{2}\right)$ & $V_{0}(\mathrm{~m} / \mathrm{s})$ \\
\hline 400 & 80 & 0 & $1 \times 10^{7}$ & 0.075 & 0.0625 & 4.5
\end{tabular}

the protected structure predicted by the present collision model and calculated by Li et al. [22]. The detailed parameters are listed in Table 1.

It can be seen in Figure 4 that the velocity attenuation of the first impact process predicted by the present model is very close to that calculated by $\mathrm{Li}$ et al. [22]. Since the friction dissipation energy is ignored, the second impacting 


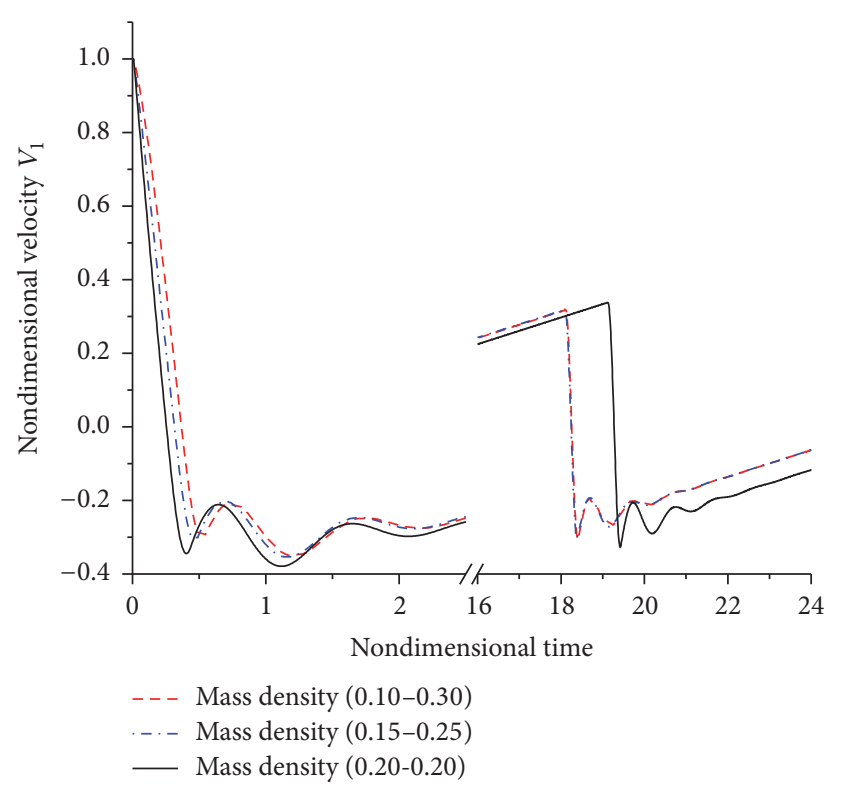

(a)

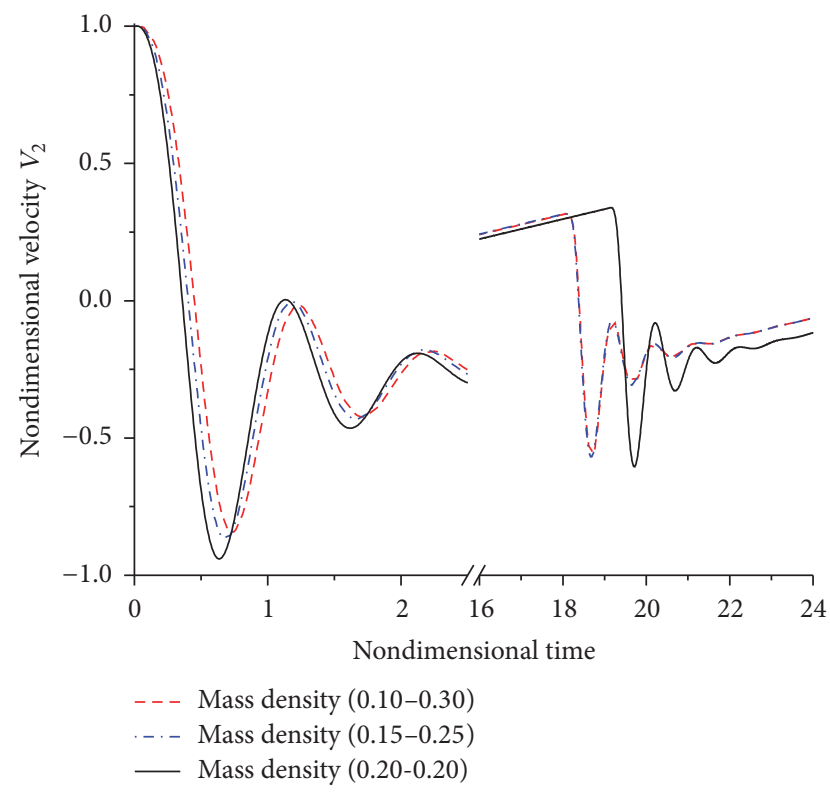

(b)

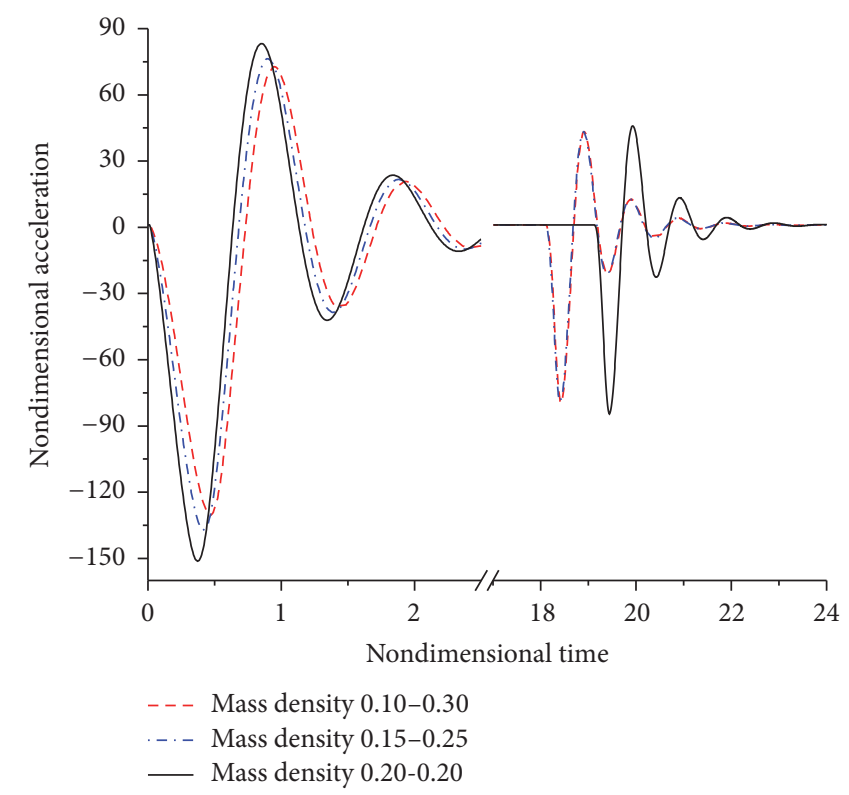

(c)

FIGURE 5: The impacting process: (a) the velocity attenuation of outer protected structure $M_{1}$; (b) the velocity attenuation of inner protected structure $M_{2} ;(\mathrm{c})$ the acceleration attenuation of inner protected structure $M_{2}$.

velocity calculated by present model is lightly larger than that calculated by Li et al. [22].

\section{Discussion}

4.1. The Influence of Gradient Cellular Material on Velocity Attenuation of Protected Structure. To compare with the homogeneous cellular material, the mass of gradient cellular materials is kept as constant. Moreover, to describe the changing range of the linear mass density distribution, the gradient change is defined as the difference between $\rho_{2}$ and $\rho_{1}$. The velocity and acceleration attenuation of protected structure are shown in Figure 5.

As the gradient change increases, the minimum mass density decreases. Consequently, the crushing thickness of the gradient cellular material with the mass density distribution (0.1-0.3) is larger than that of the others after the first impact. And the first impact duration of the gradient cellular material with the mass density distribution (0.10.3 ) is larger than that of the others. Therefore, the first rebounding velocities $V_{1}$ and $V_{2}$ and the first rebounding 


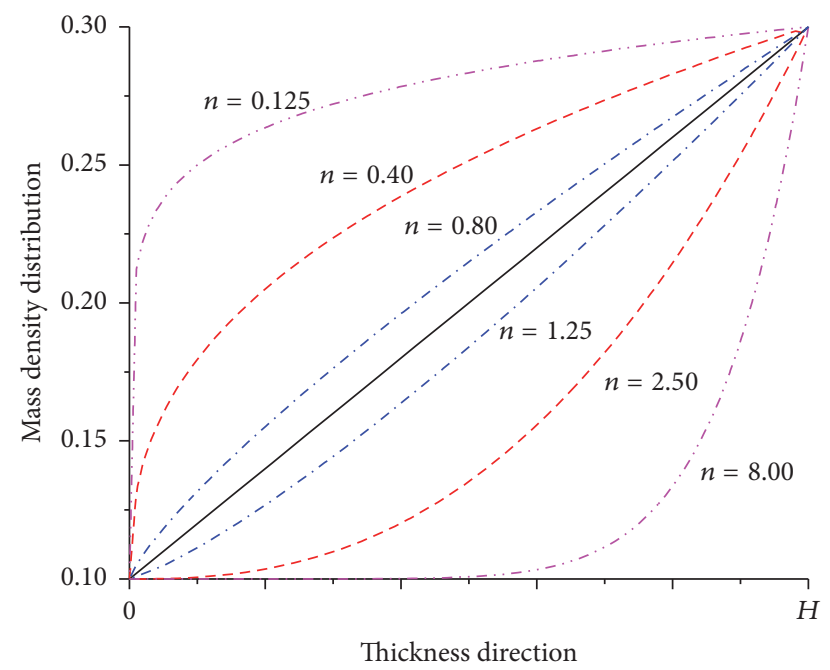

FIGURE 6: The nonlinear mass density distribution of gradient metallic cellular material along thickness direction.

acceleration $a_{2}$ decrease as the gradient change increases in Figure 5. Meanwhile, it can be concluded that adopting gradient cellular materials can effectively reduce the maximum acceleration value of inner protected structure during the first impact compared with homogeneous cellular materials with the same mass.

4.2. The Influence of Nonlinear Mass Density Distribution of Gradient Metallic Cellular Material on the Acceleration Attenuation of Inner Protected Structure. In this section, the mass density distribution of gradient metallic cellular material is calculated as follows:

$$
\rho_{\text {initial }}(z)=\rho_{1}+\left(\rho_{2}-\rho_{1}\right) \cdot\left(\frac{z}{L}\right)^{n},
$$

where the gradient index $n$ is separately equal to $0.125,0.40$, $0.80,1.25,2.5$, and 8.0 . The nonlinear mass density distribution along thickness direction is shown in Figure 6. And the influence of mass density distribution on the maximum acceleration of inner protected structure $M_{2}$ during the first impacting process is shown in Figure 7.

It can be seen that the maximum acceleration value of inner protected structure $M_{2}$ after the first impact decreases as the value of gradient index $n$ increases in Figure 7. When gradient index $n$ is more than 2.5, the mass densities near the upper face gradually keep the same shown in Figure 6. Therefore, the crushing thicknesses also gradually keep the same. And the maximum acceleration value decreases less after the first impact though the gradient index $n$ increases as in Figure 7.

\subsection{The Influence of the Spring on Acceleration Attenuation of} the Inner Protected Structure. During the impact, the kinetic energy of the inner protected structure can be absorbed by the spring and then transferred partially to the outer structure. The influence of spring on acceleration attenuation of inner protected structure is shown in Figure 8.

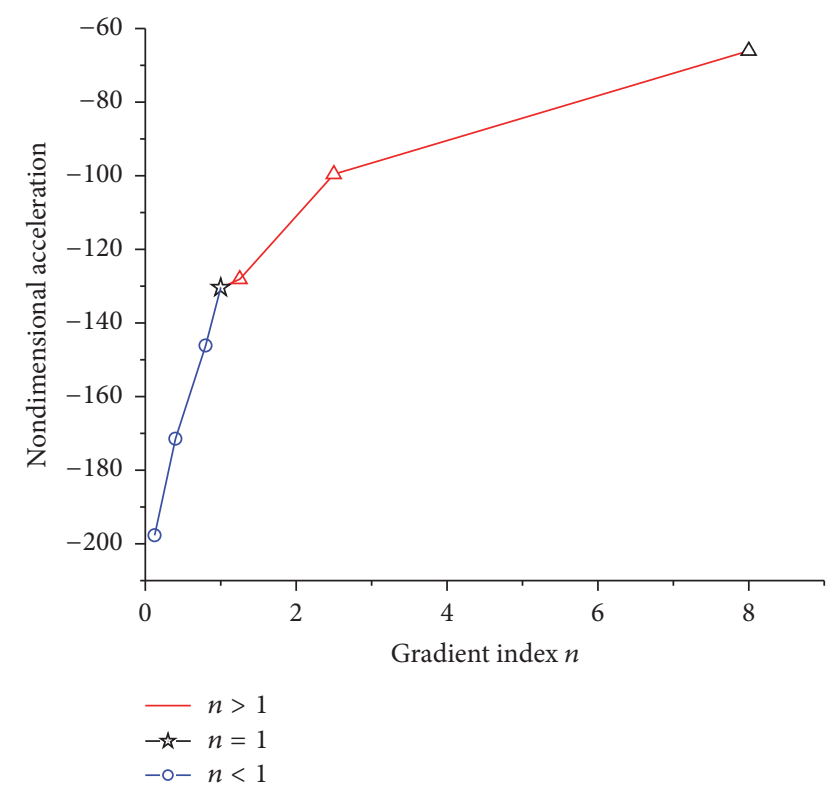

FIgURE 7: The influence of mass density distribution on the maximum acceleration value of inner protected structure $M_{2}$.

It can be seen in Figure 8(a) that the maximum acceleration value of inner protected structure decreases as the initial stiffness value $k_{0}$ decreases. This is because more kinetic energy of inner structure has been stored in spring and absorbed by gradient cellular material eventually. In Figure $8(\mathrm{~b})$, the maximum acceleration value of inner protected structure during the first impacting process slightly increases while the moment of the second impact delays as $k$ increases. Therefore, to decrease the maximum acceleration value of inner protected structure during the first impacting process, the initial stiffness value of the spring should be chosen carefully.

\section{Conclusion}

(1) A formula associated with hardening strain for cellular material is presented to predict the crushing stress of gradient metallic cellular material under quasistatic compression. The FE result shows that this formula can predict the crushing stress of the gradient metallic cellular material.

(2) A collision model for protective structure with gradient metallic cellular material under low velocity impact is established, in which the gradient metallic cellular material is used for protection. The result shows that adopting the gradient metallic cellular material instead of homogeneous cellular material can effectively decrease the maximum acceleration value of inner protected structure during the first impacting process. 


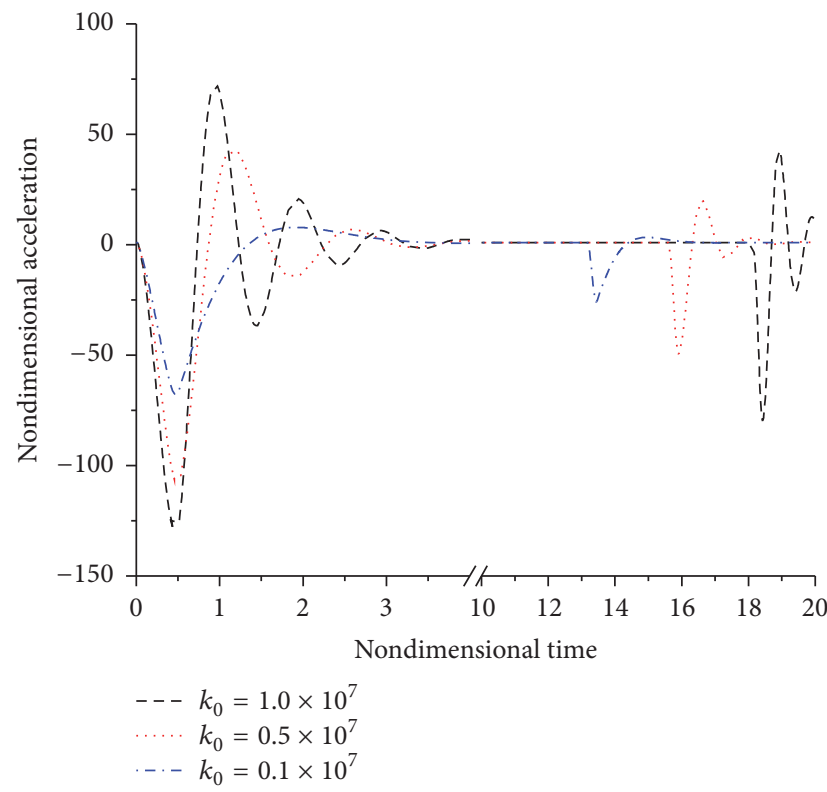

(a)

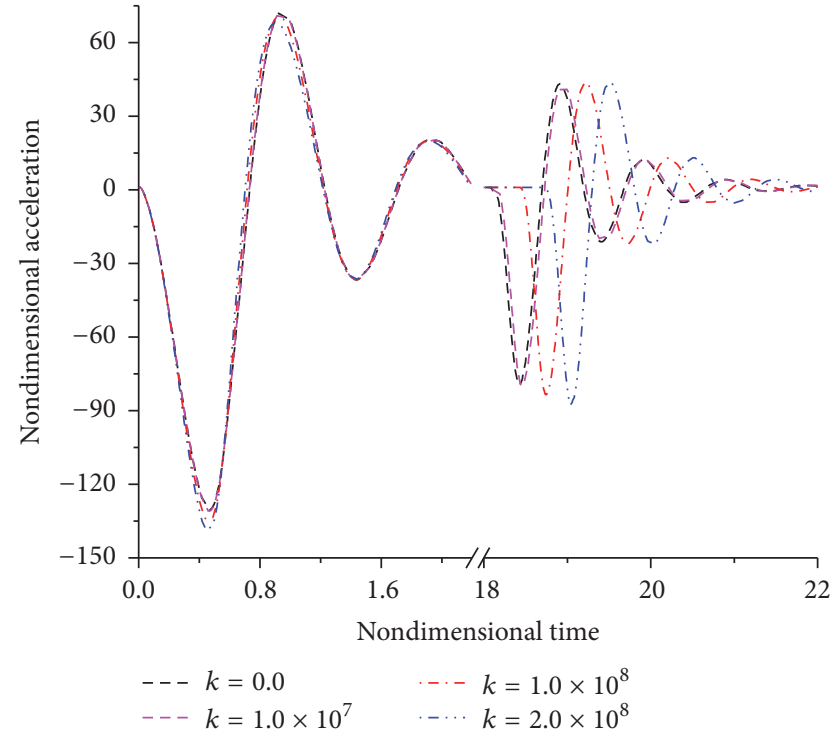

(b)

FIGURE 8: The acceleration attenuation of inner protected structure $M_{2}$ : (a) $k_{0}$ increases when $k=0$; (b) $k$ increases when $k_{0}=1 \times 10^{7}$.

\section{Competing Interests}

The authors declare no potential competing interests with respect to the research, authorship, and/or publication of this article.

\section{Acknowledgments}

This work is supported by the National Natural Science Foundation of China (11372237).

\section{References}

[1] H. B. Zeng, S. Pattofatto, H. Zhao, Y. Girard, and V. Fascio, "Perforation of sandwich plates with graded hollow sphere cores under impact loading," International Journal of Impact Engineering, vol. 37, no. 11, pp. 1083-1091, 2010.

[2] X. R. Liu, X. G. Tian, T. J. Lu, D. Q. Zhou, and B. Liang, "Blast resistance of sandwich-walled hollow cylinders with graded metallic foam cores," Composite Structures, vol. 94, no. 8, pp. 2485-2493, 2012.

[3] X. R. Liu, X. G. Tian, T. J. Lu, and B. Liang, "Sandwich plates with functionally graded metallic foam cores subjected to air blast loading," International Journal of Mechanical Sciences, vol. 84, pp. 61-72, 2014.

[4] S. Li, Z. Wang, G. Wu, L. Zhao, and X. Li, "Dynamic response of sandwich spherical shell with graded metallic foam cores subjected to blast loading," Composites Part A: Applied Science and Manufacturing, vol. 56, pp. 262-271, 2014.

[5] Z. Jin, C. Yin, Y. Chen, and H. Hua, "Graded effects of metallic foam cores for spherical sandwich shells subjected to close-in underwater explosion," International Journal of Impact Engineering, vol. 94, pp. 23-35, 2016.
[6] G.-D. Xu, F. Yang, T. Zeng, S. Cheng, and Z.-H. Wang, "Bending behavior of graded corrugated truss core composite sandwich beams," Composite Structures, vol. 138, pp. 342-351, 2016.

[7] D. B. Xiao, L. Mu, and G. P. Zhao, "The influence of correlating material parameters of gradient foam core on free vibration of sandwich panel," Composites Part B: Engineering, vol. 77, pp. 153-161, 2015.

[8] M. Ali, A. Qamhiyah, D. Flugrad, and M. Shakoor, "Theoretical and finite element study of a compact energy absorber," Advances in Engineering Software, vol. 39, no. 2, pp. 95-106, 2008.

[9] D. Ruan, G. X. Lu, B. Wang, and T. X. Yu, "In-plane dynamic crushing of honeycombs-a finite element study," International Journal of Impact Engineering, vol. 28, no. 2, pp. 161-182, 2003.

[10] A. Ajdari, P. Canavan, H. Nayeb-Hashemi, and G. Warner, "Mechanical properties of functionally graded 2-D cellular structures: a finite element simulation," Materials Science and Engineering: A, vol. 499, no. 1-2, pp. 434-439, 2009.

[11] Y. Hangai, K. Saito, T. Utsunomiya, O. Kuwazuru, and N. Yoshikawa, "Fabrication and compression properties of functionally graded foam with uniform pore structures consisting of dissimilar A1050 and A6061 aluminum alloys," Materials Science and Engineering A, vol. 613, pp. 163-170, 2014.

[12] S.-Y. He, Y. Zhang, G. Dai, and J.-Q. Jiang, "Preparation of density-graded aluminum foam," Materials Science and Engineering A, vol. 618, pp. 496-499, 2014.

[13] D. B. Xiao, L. Mu, and G. P. Zhao, "Indentation response of sandwich panels with positive gradient metallic cellular core," Journal of Sandwich Structures \& Materials, vol. 17, no. 6, pp. 597-612, 2015.

[14] D. B. Xiao, L. Mu, and G. P. Zhao, "Influence of positive gradient metallic cellular core on energy dissipation of sandwich panels under indentation," Archive of Applied Mechanics, 2016. 
[15] S. R. Reid and C. Peng, "Dynamic uniaxial crushing of wood," International Journal of Impact Engineering, vol. 19, no. 5-6, pp. 531-570, 1997.

[16] V. P. W. Shim, Z. H. Tu, and C. T. Lim, “Two-dimensional response of crushable polyurethane foam to low velocity impact," International Journal of Impact Engineering, vol. 24, no. 6, pp. 703-731, 2000.

[17] W. Altenhof, C. Powell, A.-M. Harte, and R. Gaspar, "An experimental investigation into the energy absorption and force/displacement characteristics of aluminum foam filled braided stainless steel tubes under quasistatic tensile loading conditions," International Journal of Crashworthiness, vol. 10, no. 1, pp. 21-31, 2005.

[18] N. K. Bourne, K. Bennett, A. M. Milne, S. A. MacDonald, J. J. Harrigan, and J. C. F. Millett, "The shock response of aluminium foams," Scripta Materialia, vol. 58, no. 2, pp. 154-157, 2008.

[19] Z. Ahmad, D. P. Thambiratnam, and A. C. C. Tan, "Dynamic energy absorption characteristics of foam-filled conical tubes under oblique impact loading," International Journal of Impact Engineering, vol. 37, no. 5, pp. 475-488, 2010.

[20] G. Joshi, A. K. Bajaj, and P. Davies, "Whole-body vibratory response study using a nonlinear multi-body model of seatoccupant system with viscoelastic flexible polyurethane foam," Industrial Health, vol. 48, no. 5, pp. 663-674, 2010.

[21] M. G. R. Toward and M. J. Griffin, "A variable parameter single degree-of-freedom model for predicting the effects of sitting posture and vibration magnitude on the vertical apparent mass of the human body," Industrial Health, vol. 48, no. 5, pp. 654662, 2010.

[22] B. C. Li, G. P. Zhao, and T. J. Lu, "A double degree freedom massspring-damper-foam collision model for high porosity metallic foams," Journal of Applied Mechanics, vol. 79, no. 5, Article ID 051021, 2012.

[23] B. C. Li, G. P. Zhao, and T. J. Lu, "Large mass protection with close-celled metallic foams under low velocity impact: spring-damper-foam collision model," International Journal of Nonlinear Sciences and Numerical Simulation, vol. 13, no. 1, pp. 39-47, 2012.

[24] B. C. Li, G. P. Zhao, and T. J. Lu, "Low strain rate compressive behavior of high porosity closed-cell aluminum foams," Chinese Journal of Theoretical and Applied Mechanics, vol. 43, no. 1, pp. 122-135, 2011.

[25] Z. J. Zheng, C. F. Wang, J. L. Yu, S. R. Reid, and J. J. Harrigan, "Dynamic stress-strain states for metal foams using a 3D cellular model," Journal of the Mechanics and Physics of Solids, vol. 72, pp. 93-114, 2014.

[26] S. L. Wang, Y. Y. Ding, C. F. Wang, Z. J. Zheng, and J. Yu, "Effect of relative density of the dynamic impact behaviors of closed cell foam," in Proceedings of the ASME 35th International Conference on Ocean, Offshore and Arctic Engineering (OMAE '16), Busan, South Korea, June 2016.

[27] L. J. Gibson and M. F. Ashby, Cellular Solids: Structure and Properties, Cambridge University Press, Cambridge, UK, 1997. 


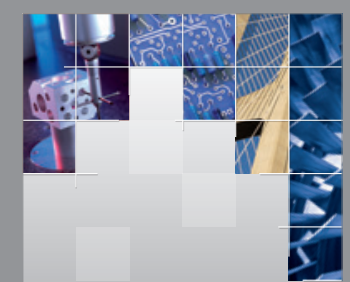

\section{Enfincering}
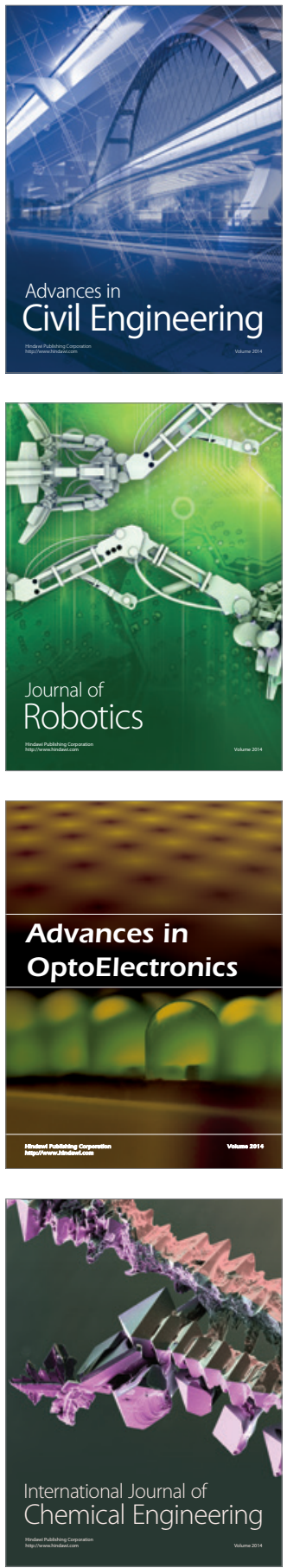

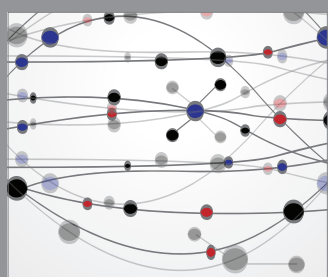

The Scientific World Journal

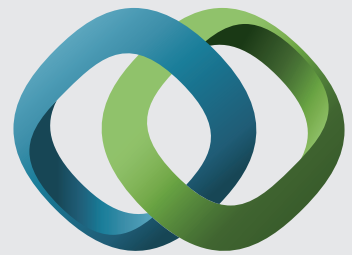

\section{Hindawi}

Submit your manuscripts at

http://www.hindawi.com
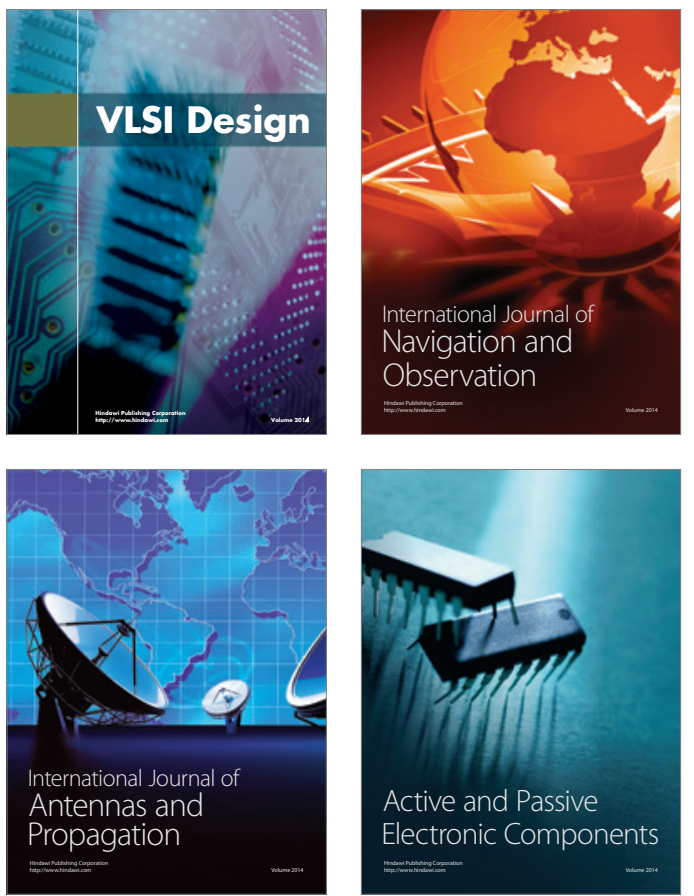
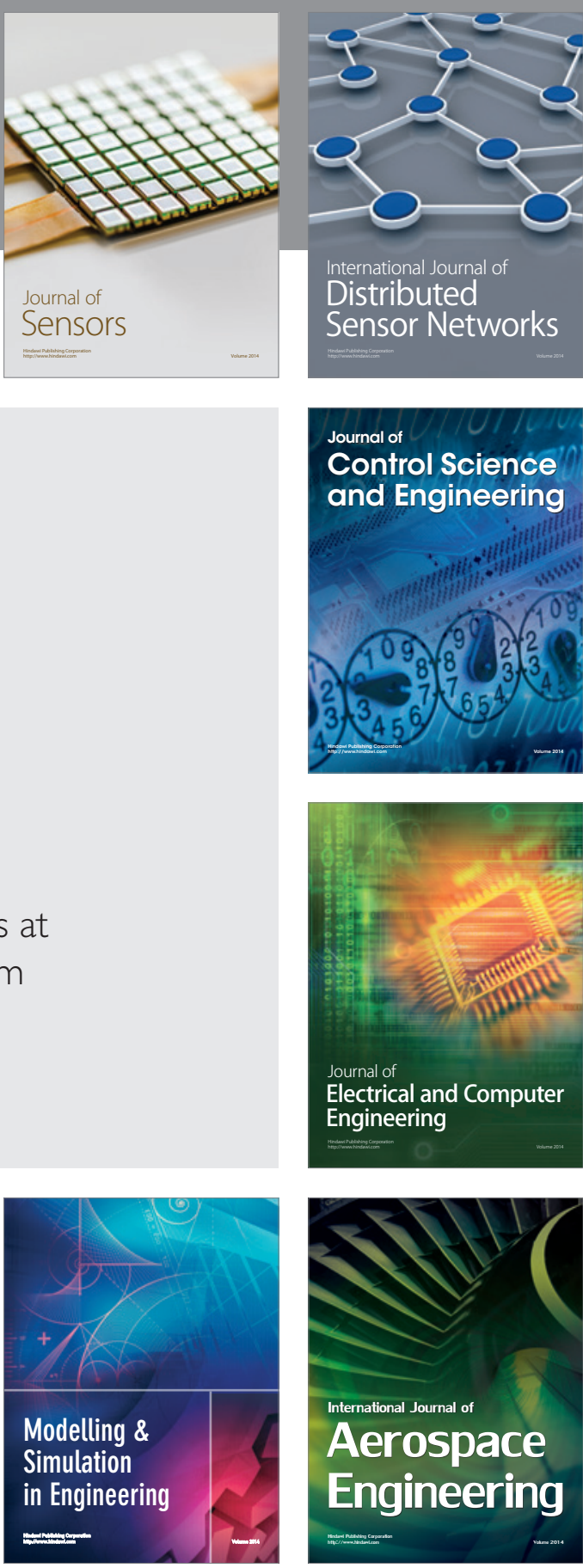

International Journal of

Distributed

Sensor Networks

Journal of

Control Science

and Engineering
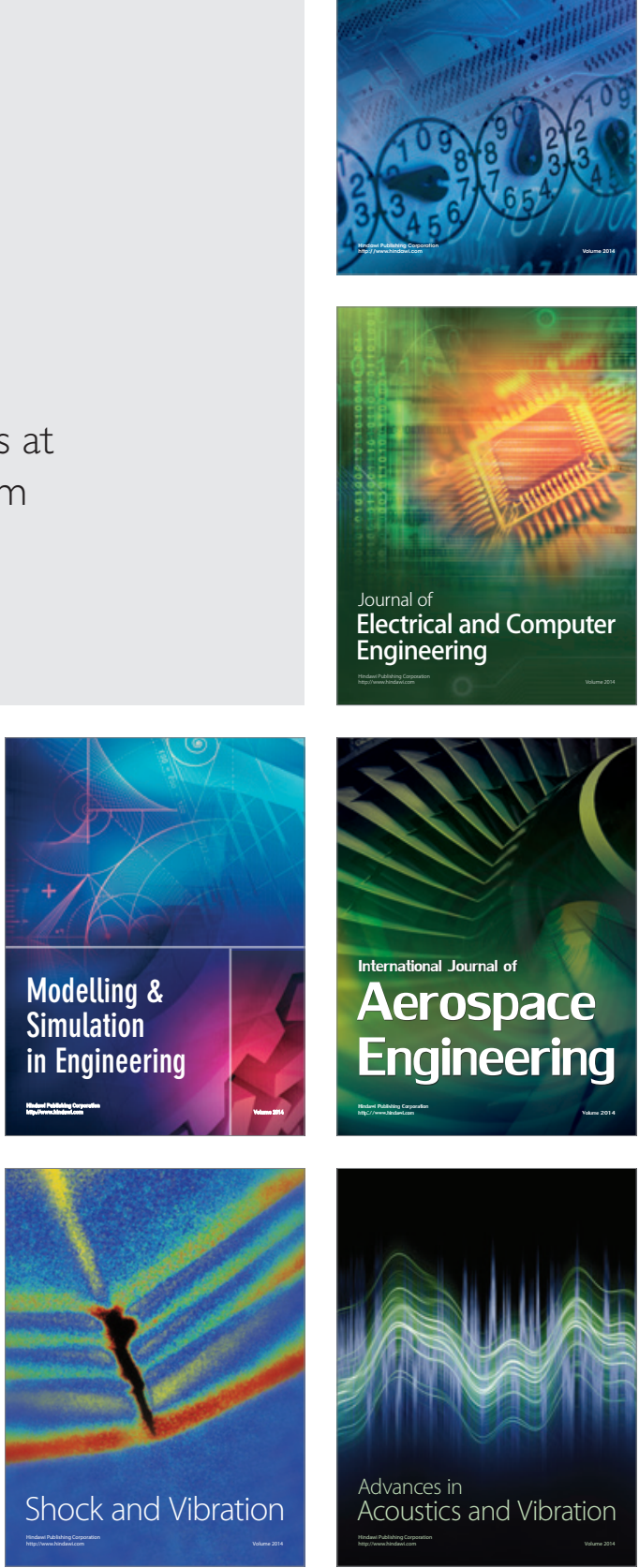